\title{
The Role of the Teacher or Knowledgeable Peer in Language Learning: Visual Scaffolding
}

\author{
Jamal A. Khlifat, MA, MSLS \\ Department of Linguistics, University of Colorado, Boulder \\ jamal.khlifat@Colorado.EDU,j.a.khlifat@eagle.clarion.edu
}

\begin{abstract}
What is the role of the teacher or more knowledgeable peer in language learning? This research examines a case study that has been conducted in the Middle East (Jordan) to examine the role of the teacher in language learning on the semantic and lexical levels. The case study targets two groups of Arabic speakers learning English. While visual scaffolding was used with Group B, Group A was not exposed to any form of visual scaffolding. The study touches on the role of "visual scaffolding" in language learning, which is the very provision of appropriate assistance, using visual aids, given to the target student to help him/her achieve something that is beyond his/her sole potential way oflearning. Based on this, the research claims that there is a gap between the learner's current level of development and that potential one that is guided by a teacher or by some peers who are more capable in this arena. This gap is often referred to as the Zone of Proximal Development (ZPD). To achieve this potential progress, input must be comprehensible. When the input is comprehensible, spectacular progress in language acquisition occurs provided that the "Affective Filter" is absent. The latter has to do with boredomand nervousness. According to the study, not only will this filter make the subject content incomprehensible, but it will also hamper learning the language itself. The study also claims that for a perfect setting of learning to happen, visual scaffolding must be present. The study concludes that this technique is very useful to kill the affective filter and make the input drastically more compreh ensible.
\end{abstract}

Keywords: scaffolding, visual scaffolding, affective filter, ZPD, language acquisition

\section{INTRODUCTION}

In the field of language learning and acquisition, learners face many obstacles as they attempt to learn the target language. These obstacles can lead to frustration and anxiety, which in the long run will hamper the learning process. The problem arises on the lexical and semantic levels since there does not seem to be one-to-one correspondence between the lexicon of one language and that of another. Every language has its own exotic and peculiar lexical dictionary that, in a way or another, satisfies some of the lexical needs of the other language. In other words, cultural barriers drastically influence the lexicon of languages. What seems to be a lexical entry of a constant use in a particular language may not be so in another language. To satisfy and to overcome some of the obstacles that arise from this fact, visual scaffolding comes handy as an effective means for helping second language learners become involved in the learning process and kill the state of anxiety and frustration that generates from understanding little or nothing in the learning environment.

\section{LITERATURE REVIEW}

Scaffolding and visual scaffolding strategies have been the domain of research in the field of applied linguistics in general and second language acquisition in particular. Researchers in the field of language acquisition strive to study the effect of scaffolding and visual scaffolding on the process of language learning. The technique has proved itself valid in motivating learners, especially those who are in the elementary phase, to make great progress in learning the target language. Proponents of this technique make use of drawings, photos, images, videos and other visual tools harnessing them to trigger motivation in their target learners to help them learn, and perhaps master, the target language with less effort and with confidence (Herrell \& Jordan, 2012). It is all about doing away with the effective filter that hampers the second language learners' competence, leading to poor performance in the practical arena. This study comes as a complementary one to those that have earlier been conducted in the field to reinforce the positive aspects of this strategy. At the same time, it comes 
handy to bring to light some of its drawbacks. This case study has adopted its basis from some renowned figures in cognitive psychology and language acquisition: Vygotsky, Krashen and Bruner.

\section{MeThodology}

For the purpose of this case study, visual scaffolding has been used in an English-as-a-second language environment to see the impact the scaffolding and visual scaffolding leave on ESL learners. The case study involved 12 subjects who were evaluated to be in the elementary level on the language-learning range. The subjects were almost in the same phase: poor English lexicon, poor grammar and poor reading and listening competence skills. The subjects were divided into two classrooms - six each. The first group was exposed to general scaffolding, provisional assistance given by an instructor without the assistance of visual images, drawings, videos and other potential visual tools that tend to ease the learning process and kill the affective filter that results from understanding nothing or very little in the class room. The second group was assisted with "visual scaffolding"; the learning process was supported with images, graphs, and drawings to help learners understand the term/ concept in question. The study targeted and tackled the semantic and lexical phase of the language. Subjects were taught vocabulary lists tackling names of fruits and vegetables. Some of these fruits and vegetables were unfamiliar to the subjects due to the fact that they do not grow in Jordan; they were even hard to come by in the market. Upon asking both groups to memorize these lists of words, students had to sit for a vocabulary test to demonstrate their learning of new vocabulary lists. The instruction time for both groups was the same, but the timing was different. The instruction sessions were held twice a week, and each session lasted 150 minutes. Upon calculating the test scores for each individual in each group, results were added up, and the means (average) was considered to evaluate the outcome of the learning process under the impact of general scaffolding and visual scaffolding.

\section{DisCUSSION}

\subsection{Results}

The very crucial goal of this case study was to analyze and examine the very positive impact, if there is any, of visual scaffolding on increasing students' vocabulary. The case study has targeted the semantic and lexical level. Upon obtaining the test scores for both groups, it was obvious that students who were exposed to visual scaffolding performed better. Students were able to match the right image to the right concept. Those who had not been exposed to visual scaffolding succeeded in matching some images to the right concepts, but they left out many, and they mismatched some. In the sections where no matching between the concept and the image and where it depended on providing a compositional description of the word in question, those who had been exposed to visual scaffolding managed to provide, to a certain degree, accurate description of the referent that the word mapped to. This was obviously due to the fact that they were exposed to the referent image in the instruction sessions; they were able to remember that referent and describe it as if it were portrayed in front of them. The average score for the exposed-to-visual-scaffolding group (Group B) was 8.5 out of 10, but it was only 7 out of 10 for the Group A-with general scaffolding only. This study will be a foundation study for another one that is planned to be held in the Middle East next summer. The future study is expected to have students from more than one dialect of Arabic, albeit in the same ESL learning stage. The number of subjects will be much larger, and subjects are expected to be divided into 3 main instruction sessions. The first is a group without any kind of scaffolding, the second group will be exposed to general academic scaffolding and the third group will be exposed to both general academic scaffolding and visual scaffolding. The purpose of this study will also address the impact of scaffolding in general and visual scaffolding in particular on students' motivation to learn the language in question.

The outcome of this study reinforces the outcome of previous studies. It has been noticed that scaffolding and particularly visual scaffolding is an effective learning strategy for novel learners, but its failure and success are largely determined by how successful it is used and implemented by practitioners. According to Herrell and Jordan (2004), there are two successful ways to scaffold instruction with second language learners: Academic language scaffolding and visual scaffolding. Visual scaffolding is the most effective on the semantic and lexical level, especially when it accompanies effective strategies in working with learners. Two stipulations are there: the first is that language must be simplified so that students can understand. The second is that abundance of visuals 
must support the instruction and learning process. Speaking to the first stipulation and based on the case study done in this regard, it is crucial that the instructor pay attention to what is called Hypo and Hyper-articulation. Hypo-speech, which is system-oriented, speaker driven, should be abandoned since its main aim is minimizing articulatory effort. This type of articulation may be adopted in more advanced conversation classes, not with novel learners. In lieu of that, instructors should strive to adopt hyper-speech strategies, which are output-oriented and listener driven since their goals are to maximize intelligibility. When such strategies are adopted, scaffolding and visual scaffolding could not be successful any further.

The latter involves any kind of support that makes use of images that can be seen as well as concepts and words that can be heard. This effective strategy is one of the best scaffolding techniques to make input comprehensible to second language learners. It definitely goes beyond making the integral subject content easy to contribute to faster and more effective acquisition of the target language on the semantic and lexical level. In this regard, the notion of the Zone of Proximal Development, coined by Lev Vygotsky, proves itself valid. ZPD is that notional gap between the learner's current level of development, when adopting learning and problem-solving strategies independently, and that level of potential development when learning and problem-solving strategies are done under the guidance and help of an adult teacher or a more capable or knowledgeable peer. The instructor's help and guidance can be best depicted as a beam of light that tends to help the learner walk out of his/her current zone of development. This is due to the fact that when learners are in their own zone of development, meaning learning the input independently, the input may not be fully comprehensible, and a more knowledgeable peer's or instructor's guidance is needed to achieve this goal. When input is made comprehensible, sufficient useful milieu for language learning is made available.

But even when general scaffolding is there and a certain degree of comprehensible input is there, the affective filter might hamper the language learning process. In fact, it is almost impossible to learn a language under the influence of the affective filter. The affective filter is that filter that is caused by fear, nervousness, boredom and lack of interest in the subject matter due to understanding very little or nothing at all. This suggests that sometime this kind of general, pure oral scaffolding, although it contributes to make the input comprehensible, it is neither sufficient to outstanding progress in language learning, nor is it so to kill and uproot the affective filter. This is where visual scaffolding comes handy; learners benefit the most from any type of scaffolding that makes extensive implementation of visual aids. The whole idea resides in the fact that seeing the image of what the instructor or more knowledgeable peer is describing or hearing the key word of what the guide (the instructor or the peer) is explaining will considerably make the input more comprehensible. It will also, in the long run, help the learner kill and do away with the affective filter that usually generates from the fear and boredom that are triggered due to assimilating very little or nothing at all in class.

The question that raises itself for the moment is that, How far can we depend on visual scaffolding? Well, we should be rational to accept the fact that visual scaffolding has its own drawbacks; it does seem limited to certain levels of instruction. Visual scaffolding is most effective on the semantic and lexical levels, but even in this regard, there are some limitations.

The problem arises from the fact that not all lexical words/ concepts refer to concrete observable referents. When the word has a concrete observable referent, visual scaffolding comes handy since it depicts and portrays this concept in a form of some kind of visual aids to make the input comprehensible. Every language has its own exotic mental dictionary (lexicon). When the denotation of the concept is needed and this concept refers to a concrete observable referent, visual scaffolding does the job, but when the connotation is needed to make the input comprehensible, visual scaffolding will fall short to satisfy this requirement. Some words have their own expressive meaning that only exist in the target language, and visual scaffolding cannot help express and explain this meaning. This is true since meaning is all about the relationship of the word to the object it represents and/or the concept it explains. The is due to the fact that applying a word requires us to look up the word in our mental dictionary, matching the thing in the environment that best matches that and then mapping the word to the referent it represents. Although visual scaffolding can contribute greatly to the learning process in this regard, the fact that not all objects so easily match to words is a challenging problem in this arena. In other words, some instances or uses of the word do not match the real objects. The following sentences make use of the word "mouse," but none of them means the usual organism "known as a mouse": 
- Donna is no mouse

- I am sure he will attempt to make a pass at that little mouse over there

- He does not definitely want those mice any deader than they were

- I am just wondering if he is a man or a mouse

Each one of these sentences has the word "mouse" in it, but they appear to have different meanings from what the actual usage of the word refers to; no one of them refers to a small rodent with big ears, a long thin tail and a long thin snout. What is intriguing about such uses is that they are really common, and such uses sweep and overrun what the real denotation of the word is. It seems appropriate to hypothesize here that the lexical term is merely a relationship between sound/ movement and concept while the real word is left abandoned.

Another problem arises from the notion of abstract notions; it is well-known that not all lexical items (words) refer to real concrete objects in the world. Abstract notions such as joy, beauty, abstinence and cognition cannot be made comprehensible by the mere usage of visual scaffolding. How can the teacher visually scaffold instruction to explain these concepts? The issue becomes more complicated when consider that challenging subtype called "verbs." Although visual scaffolding proves itself, to a certain extent, helpful and beneficial in making action verbs comprehensible input, it is not at all when we consider stative verbs as well as light verbs. How can an instructor or more knowledgeable peer explain the meaning of the light verb "have" in a sentence like "he is having a shower" or the meaning of the stative verb "know" in the sentence "I know this"?

\section{CONClusion}

It seems to be appropriate to conclude that visual scaffolding is very helpful in the process of language learning, particularly on the lexical and semantic levels. It helps learners increase their lexicon. Language instructors should implement this tool to make input comprehensible, especially with preliminary-learning-phase students. Based on the case study discussed here as well as previous research in the field of language learning and acquisition, language learners seem to benefit a lot from visual scaffolding. This technique has its supremacy over general scaffolding. It is an effective method that can be adopted to kill the affective filter. Although this technique comes handy in making input, to a certain degree, compressible, it is challenged by abstract notions, stative verbs, and light verbs. How can visual scaffolding overcome these obstacles?

\section{REFERENCES}

Benson, B. (1997). Scaffolding (Coming to Terms). English Journal, 86(7), 126-127.

Fox, Barbara. "Welcome to semantics and pragmatics." Lecture on English Semantics and Pragmatics, Boulder, CO, January 13, 2015.

Gillen, J. (2000). Versions of Vygotsky. British Journal of Educational Studies, 48(2), 183-198.

Hausfather, S. (1996). Vygotsky and schooling: Creating a social context for learning. Action in Teacher Education, 18(2), 1-10.

Herrell, A. L., \& Jordan, M. (2012). 50 strategies for teaching English language learners $\left(4^{\text {th }}\right.$ ed.). Boston, MA: Pearson.

Lipscomb, L., Swanson, J., West, A. (2004). Scaffolding. In M. Orey (Ed.), Emerging perspectives on learning, teaching, and technology. Retrieved on February 17, 2015 from http://epltt.coe.uga.edu/

Shoebottom, P. (n.d.). Scaffolding: An overview- ESL workshop: scaffolding theory. Retrieved on February 17, 2015 from http://esl.fis.edu/teachers/fis/scaffold/page1.htm

Vygotsky, L.S. (1978). Mind in society. Cambridge, MA: Harvard University Press. 


\section{AUTHOR'S BIOGRAPHY}

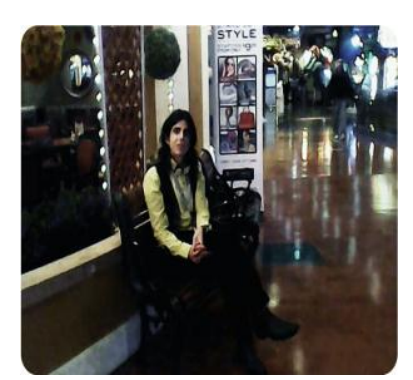

Jamal A. Khlifat, was born with a malleus in my ear enabling me to distinguish the different sounds exist in the disputed number of the world's languages. I am into anatomizing those abstract units called phonemes to examine the phonetic variations that can be observed. There is nothing as intriguing as tracing the different forms a spoken message exists in its progress from the speaker's mind to the listener's. How this peculiar linguistic signal is converted into an acoustic one, which is, in turn, converted into an electro-chemical signal in the brain, to be later on decoded into segments; words; and, ultimately, meaning, reveals the supernatural power that has created this physiological linguistic apparatus.

Research Interest: forensic linguistics, phonetics, phonology, documentation of the world's endangered languages, pharyngealization and pharyngeal harmony in Levantine Arabic, gemination in Levantine Arabic, nasalization and nasal geminates in Levantine Arabic, syntax, lexical semantics, prosody, and rhetoric and Toulmin argumentation 